\title{
A CONCEPTUAL PROJECT OF A DEVICE FOR HUMAN WRIST FUNCTIONAL REHABILITATION
}

\author{
B. LEWANDOWSKI", M. OLINSKI, S. WUDARCZYK and A. GRONOWICZ \\ Wroclaw University of Science and Technology \\ Faculty of Mechanical Engineering \\ Department of Biomedical Engineering, Mechatronics and Theory of Mechanisms \\ ul. Łukasiewicza 7/9, 50-371 Wrocław, POLAND \\ E-mail: bogusz.lewandowski@pwr.edu.pl
}

\begin{abstract}
In the paper, the problems of devices supporting functional rehabilitation of a human wrist were addressed. A literature review and a description of selected devices together with an indication of their advantages and disadvantages were conducted. The biomechanical structure of a human wrist was analyzed. On this basis and after taking into consideration ranges of motion of the selected joints the concept of a new mechanism was developed. A 3D model of the device was built in the Autodesk Inventor system. For the purpose of simulations another model was developed in the MSC Adams system. Issues of drives and sensors selection, as well as requirements for the control system, were examined.
\end{abstract}

Key words: rehabilitation support, robotic-assisted treatment, wrist rehabilitation.

\section{Introduction}

Rehabilitation is a very important aspect of human wrist dysfunctions treatment. It is intended for people who have lost some motor function due to a disease, injury, or their functions were limited due to birth defects. Rehabilitation helps to avoid potential complications associated with eventual surgery. The effectiveness of physiotherapy is significantly increased by the usage of dedicated supporting devices, which also contributes to a reduction of rehabilitation time and greatly enhances the final results. Research concentrating on increasing the effectiveness of rehabilitation has been carried out in many centers, among others in Germany, basing on the pioneering works at MIT [1, 2], where the Bi- Manu -Track system [3] was invented and implemented. It assists the exercises of pronation / supination of the forearm, as well as palmar and dorsal flexion of the wrist. Another solution, which unlike the Bi-Manu-Track has the ability to perform adduction and abduction rehabilitation movements, is the wrist unit of the MIT-Manus module. What is more, it enables the exercises of flexion and extension of the wrist, as well as the pronation and supination of the forearm. Other systems supporting rehabilitation that can reconstruct almost every movement of the upper limb are generally called exoskeletons [6-8]. One of them is named WREX and it was initially designed for children rehabilitation, but then resized for adults. It renders it possible to exercise the basic movements associated with daily functioning (reaching, eating, and washing). The main disadvantage of exoskeleton solutions is the significantly complicated mechanism, which results in their very high price. With regard to those features, the ADL system was invented [9]. It offers a wide range of basic activities, such as: opening doors, brushing teeth, etc. With three degrees of freedom, it enables the patient to exercise palmar and dorsal flexion, adduction and abduction of the wrist, pronation and supination of the forearm. There are three modes available: active, passive, and resistive. The exercises are conducted using feedback from the virtual environment, which improves the realism of tasks. The main advantage of this system is the low price and simplicity. The analysis of structures and functionality provided by the existing rehabilitation

\footnotetext{
* To whom correspondence should be addressed
} 
supporting systems leads to the conclusion that the purposeful direction of research is the design of a modular system for the upper limb. This will allow the use of each of the modules individually or properly coupled in a comprehensive system. One element of such a system may be a module for wrist rehabilitation, the idea of which is presented in this work.

\section{Design assumptions}

The first stage was to develop design assumptions, based on the current needs and requirements for this type of devices. One of the main goals was to ensure the safety of the patient. Furthermore, the whole mechanism should allow rehabilitation in human wrist's full range of motion. In addition, the designed mechanism should have a modular structure. This means that each of its modules should be able to act as a separate device supporting rehabilitation and to operate together with other modules. Details concerning the design assumptions are presented in Tab.1.

Table 1. Detailed design assumptions.

\begin{tabular}{|c|c|}
\hline Device feature & Execution \\
\hline $\begin{array}{l}\text { Provide the ability to rehabilitate movements: } \\
\text { - adduction and abduction, } \\
\text { - dorsal and palmar flexion, } \\
\text { - pronation and supination }\end{array}$ & $\begin{array}{l}\text { Design compatible with implementation of the full } \\
\text { range of motion for provided movements }\end{array}$ \\
\hline Ensuring the safety of rehabilitated people & $\begin{array}{l}\text { - } \text { series of tests and simulations on virtual and real } \\
\text { objects, } \\
\text { - maximum torques chosen within limits safe for } \\
\text { patients, } \\
\text { - safety features - mechanical and electronical }\end{array}$ \\
\hline $\begin{array}{l}\text { - The possibility of rehabilitating single joints, } \\
\text { as well as obtaining complex movements. } \\
\text { - Expandable device, } \\
\text { - Ability to adjust to every hand size (child or } \\
\text { adult), }\end{array}$ & $\begin{array}{l}\text { The construction consists of three modules: } \\
\text { - } 2 \text { providing the required movements, } \\
\text { - } 1 \text { control module with data transmitting } \\
\text { Each of the two components, providing the required } \\
\text { movements together with the control module can } \\
\text { operate as a separate rehabilitation device. }\end{array}$ \\
\hline $\begin{array}{l}\text { The axes of rotation of the device should be } \\
\text { compatible with the natural axes of the human } \\
\text { wrist }\end{array}$ & - exoskeleton type of the device \\
\hline
\end{tabular}

\subsection{Kinematic model of the structure of an upper limb}

The next stage of the design was to identify and simplify the mechanical structure of the upper limb. The axes of rotation for the wrist and forearm were presented (Fig.1) and specified rotational joint movements, that were intended to be used for supporting the rehabilitation process, were determined. After that, the forearm and wrist joints were analyzed, indicating their functions, degrees of freedom and ranges of motion. A simplified description of the properties mentioned above is shown in Tab.2. 


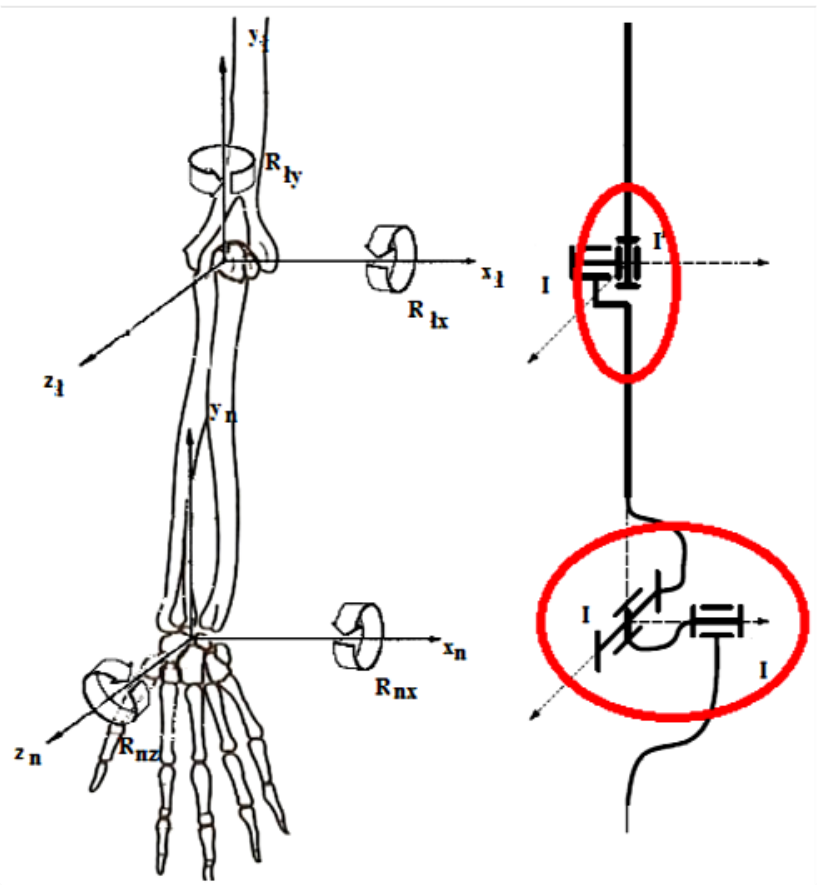

Fig.1. Simplified mechanical structure of an upper limb.

Table 2. A simplified description of structure for the forearm and wrist [10].

\begin{tabular}{|c|c|c|c|c|}
\hline $\begin{array}{c}\text { An upper } \\
\text { limb element }\end{array}$ & Joint & Description & $\begin{array}{l}\text { Number } \\
\text { of DOF }\end{array}$ & Function \\
\hline Forearm & $\begin{array}{l}\text { articulatio } \\
\text { cubiti }\end{array}$ & $\begin{array}{l}\text { Composed of three } \\
\text { joints covered by the } \\
\text { common articular } \\
\text { capsule }\end{array}$ & 2 & $\begin{array}{c}\text { Bending and straightening } \\
\text { movements (the axis of rotation is } \\
\text { not constant), and rotary motion } \\
\left(120^{\circ}-140^{\circ}\right)\end{array}$ \\
\hline \multirow[t]{2}{*}{ Wrist } & $\begin{array}{c}\text { articulatio } \\
\text { radiocarpea }\end{array}$ & $\begin{array}{l}\text { It combines a series } \\
\text { of radial bone and } \\
\text { proximal carpal } \\
\text { bones }\end{array}$ & \multirow[t]{2}{*}{2} & \multirow{2}{*}{$\begin{array}{l}\text { Palmar flexion movements }\left(50^{\circ}\right. \\
\left.\text { proximal joint, } 30^{\circ} \text { further joint }\right), \\
\text { dorsal }\left(25^{\circ} \text { proximal joint, } 45^{\circ}\right. \\
\text { further joint }) \text { and radial abduction } \\
\left(20^{\circ} \text { moving a series of bones in the }\right. \\
\text { proximal elbow direction and the } \\
\left.\text { opposite direction } 45^{\circ}\right)\end{array}$} \\
\hline & $\begin{array}{c}\text { articulatio } \\
\text { mediocarpea }\end{array}$ & $\begin{array}{l}\text { Joint of further hand, } \\
\text { created by two rows } \\
\text { of carpal bones }\end{array}$ & & \\
\hline
\end{tabular}

\section{Model of the device's structure}

Based on the analysis of the range of motion, the structure of an upper limb and the requirements of this type of devices, a conceptual model in Inventor was built. Several variants of this system were elaborated. It consists of three modules:

- $\quad$ pronation/supination of the forearm - $R_{l}$ rotation,

- $\quad$ adduction/abduction and dorsal/palmar flexion $-R_{2}, R_{3}$ rotations,

- control system module. 


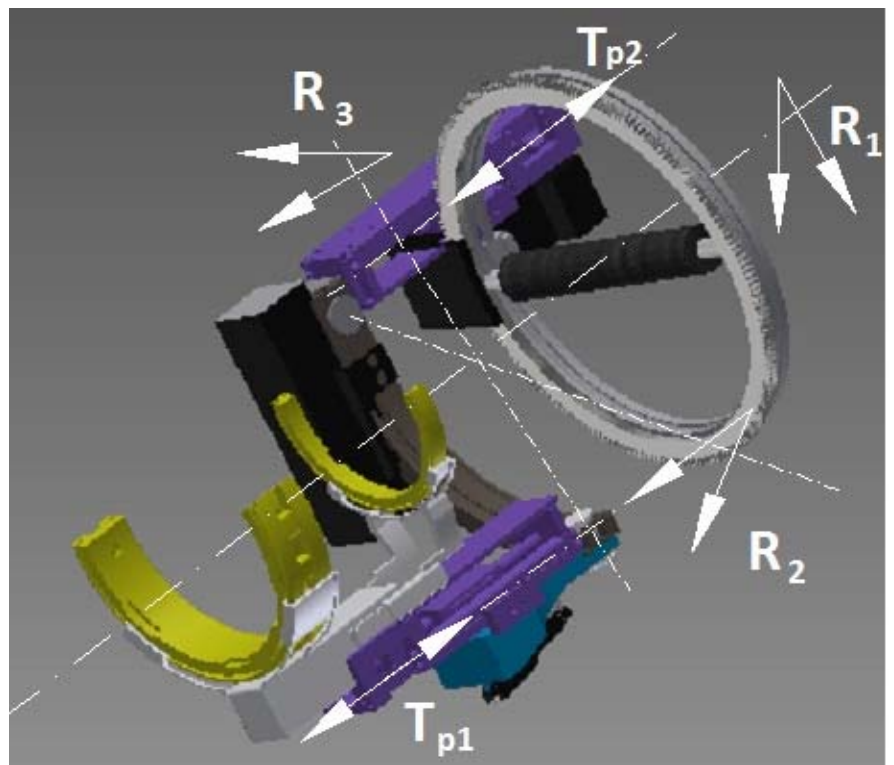

Fig.2. The model of a device supporting wrist rehabilitation: $R_{1}, R_{2}, R_{3}$ - rotations of the device; $T_{p 1}, T_{p 2}-$ adjustable movements.

The designed device was presented in a configuration composed of all the modules (Fig.2). Rotations $R_{1}, R_{2}$, and $R_{3}$ correspond to movements of pronation / supination of the forearm, dorsal and palmar bending, adduction and abduction of the wrist. In order to fit every hand, the possibility of two translational motions usage was ensured: $T_{p 1}$ and $T_{p 2}$.

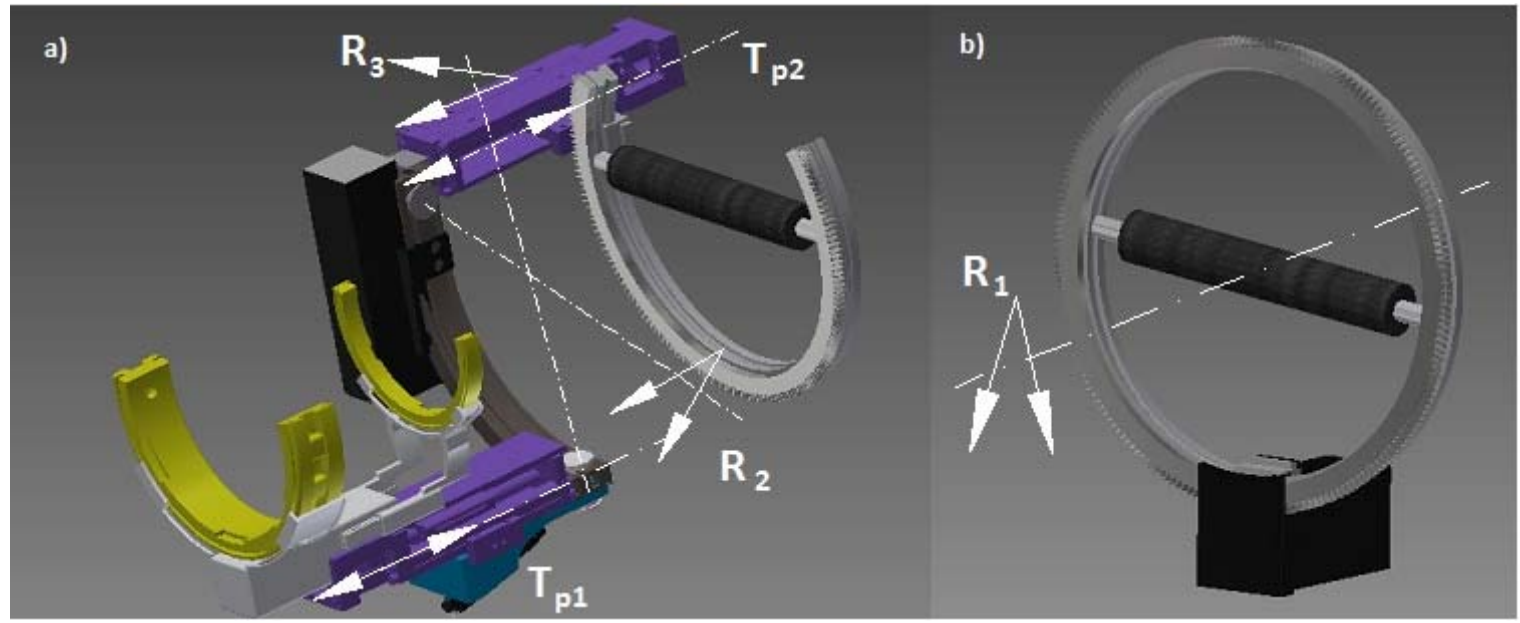

Fig.3. The model of a device supporting wrist rehabilitation: a) dorsal/palmar bending, adduction/abduction of the wrist module, $b$ ) pronation and supination of the forearm module.

Two modules were elaborated: the dorsal/palmar bending and adduction/abduction of the arm module (Fig.3a), as well as pronation and supination of the forearm module (Fig.3b). The main advantage of the idea of modular structure is the ability to perform two different parallel treatments, that do not involve movements of pronation and supination. As a result, two patients will be able to practice being supervised by a single physiotherapist. 


\section{Simulation model of the device}

For the purpose of simulation research, a model in the MSC Adams system was created. Each element has been designed taking into account the specified geometry and mass. In order to simulate the exercises using the presented model, functions describing motions were defined.

Initial conditions were determined as follows:

- At the beginning of the movement : $f=0, \dot{\phi}=0, \ddot{\phi}=0$,

- At the required range: $f=f_{\text {range }}, \dot{\phi}=0, \ddot{\phi}=0$,

where: $f$ is a general angle corresponding to $R_{1}, R_{2}, R_{3}$.

According to those assumptions, the motion was described. Constants of integration and the amplitudes were determined. Final equations describing motions are

$$
\phi(t)=\phi_{\text {range }}\left(\frac{t}{T}-\frac{1}{2 \pi} \sin \left(\frac{2 \pi}{T} * t\right)\right)
$$

where: $T$ - time of the movement cycle, $f_{\text {range }}$ - angle range for specified movement.

Thanks to the program's simulation environment it is possible to examine the dynamics of the system, including determining the loads on each kinematic pair and the drives torque demand. The most burdened kinematic pairs are the dorsal/palmar bending joint $R_{2}$ and adduction/abduction joint $R_{3}$. Loads and forces acting on the system, with the selected acceleration of individual segments, were also examined. The data may serve as a reference to determine the possibly lightest construction of the mechanism in the view of its subsequent application as a part of an exoskeleton.

The system supporting rehabilitation was invented in such a way as to avoid usage of additional sensors. A control system will be based on the analysis of data from the encoders. However, simulation results proved that it is not possible to ensure the safety of the patient without measuring the input force. Therefore a special grip construction was developed.

\subsection{Special grip module}

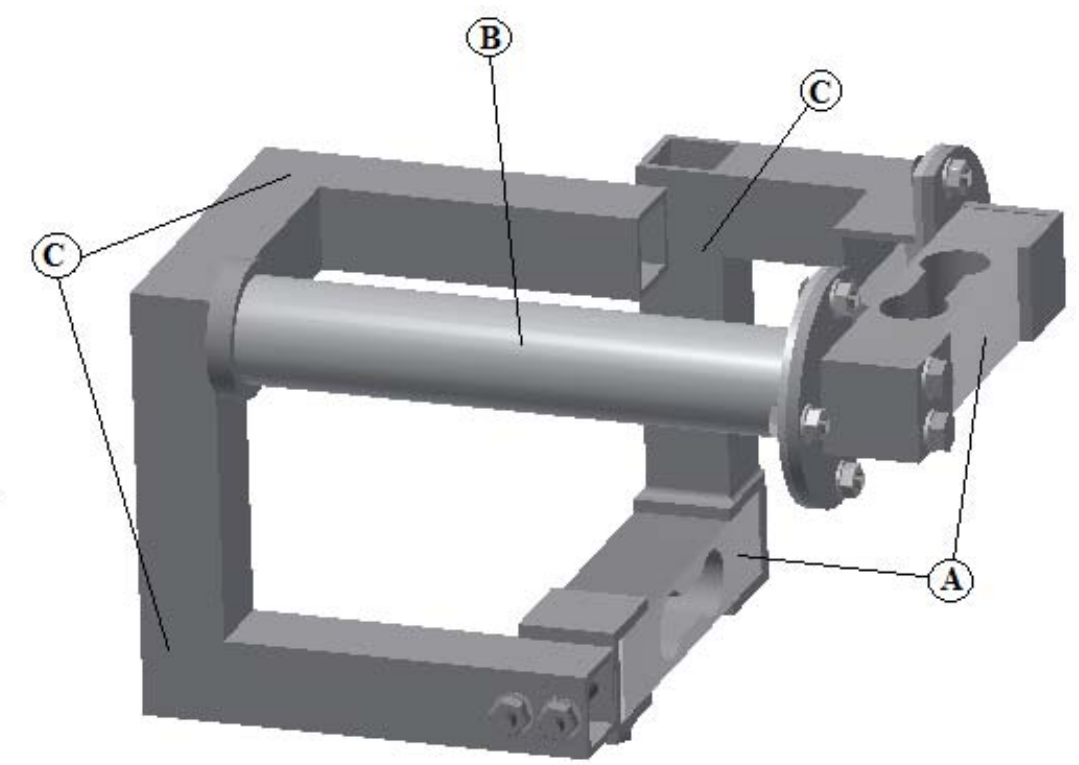

Fig.4. A special grip module - ensuring measurement of input force: A- force sensors, B- grip of the device, C- structural element. 
The construction consists of force sensors (Fig.4. A), which are located in such a way as to measure forces perpendicular to each other and construction elements (B and C), to ensure a safe grip and enough space for the human hand. To avoid disintegration of the elements they were connected via a spring. The correctness of the concept was tested in a computer simulation environment.

\subsection{Simulation research}

To prove the concept of the input force measuring module, two models were made in the MSC Adams system. The goal was to determine the force vector having known input force. The first model was based on contact forces between two solids. Unfortunately, this way of testing the device proved not to be effective due to vibrations of the system.

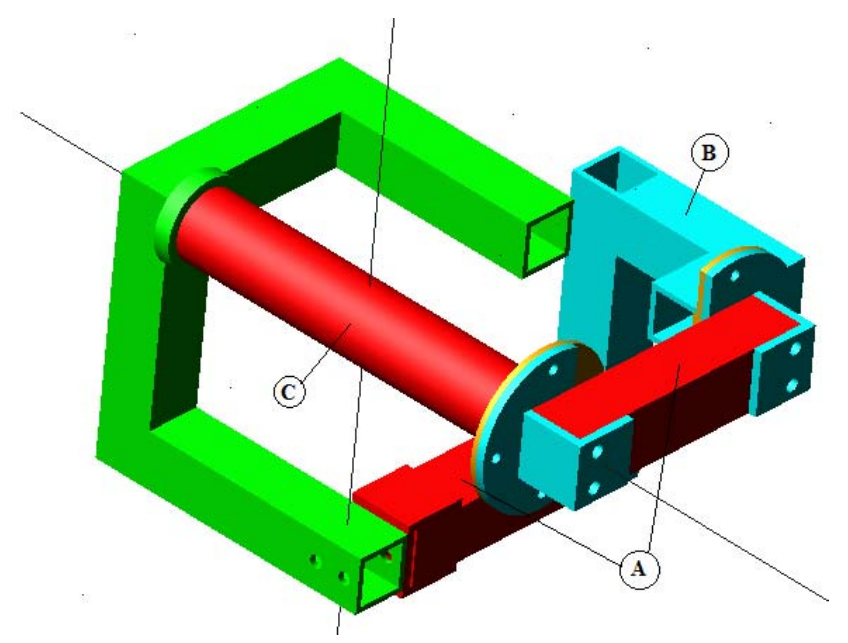

Fig.5. Input force measuring model (A- flexible beams, B-force imput part, C- grip of the device).

In order to resolve this problem, a new model was introduced (Fig.5). The sensors in this case were flexible elements (Fig.6) connected to the grip. A known input force was applied at the end of the mechanism to simulate resistance of the patient.

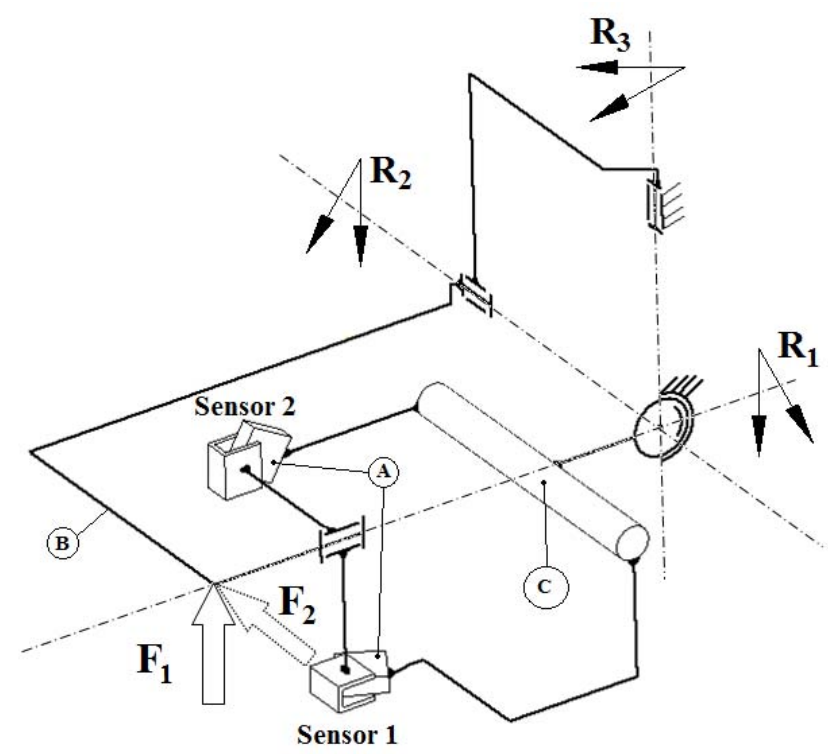

Fig.6. Input force measuring device structure: A - sensors (flexible beams), B - force input part, C- grip of the device; $F_{1}, F_{2}$ - known input forces; $R_{1}, R_{2}, R_{3}$ - supported rotations. 
Research showed (Fig.7) that the input force $F_{1}$ or $F_{2}$ of $50 \mathrm{~N}$ in single axis direction gives the response on sensors only in that axis. However, the response value is different due to different lever of acting force. It is possible due to the fact that sensors can move freely in specific directions not to disturb each other (e.g., if $F_{2}=0$, Sensor 2 can move freely in $F_{1}$ direction, in this case all the force is measured by Sensor 1). Furthermore, it gives the possibility to distinguish the force direction.

a)

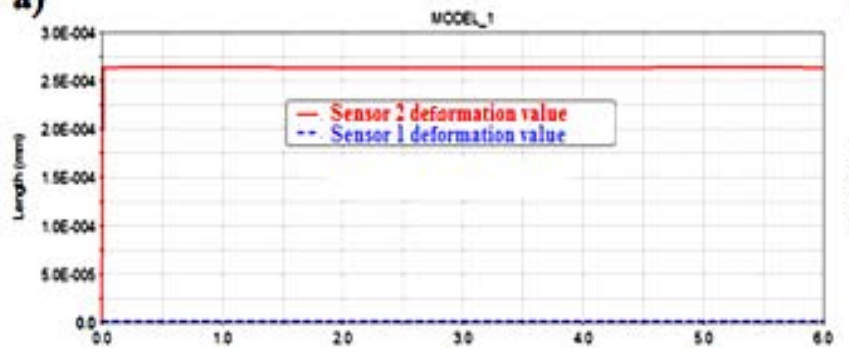

b)

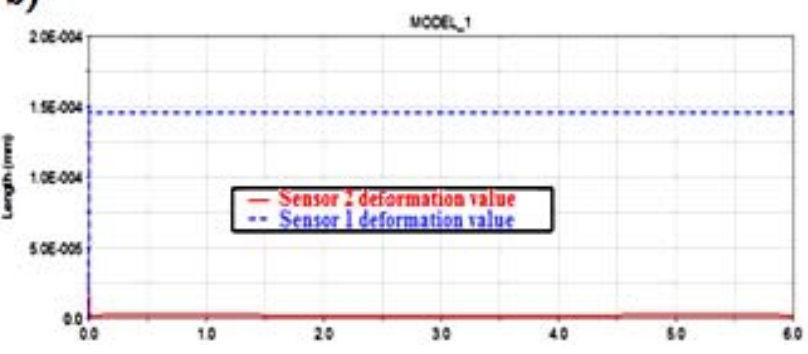

Fig.7. Sensor reading from simulations: a) $F_{1}=0, F_{2}=50 \mathrm{~N}$; b) $F_{1}=50 \mathrm{~N}, F_{2}=0$.

Research showed (Fig.8) that the input force of $50 \mathrm{~N}$ in single axis direction gives the response on sensors only in that axis. However the response value is different due to different arm of acting force.

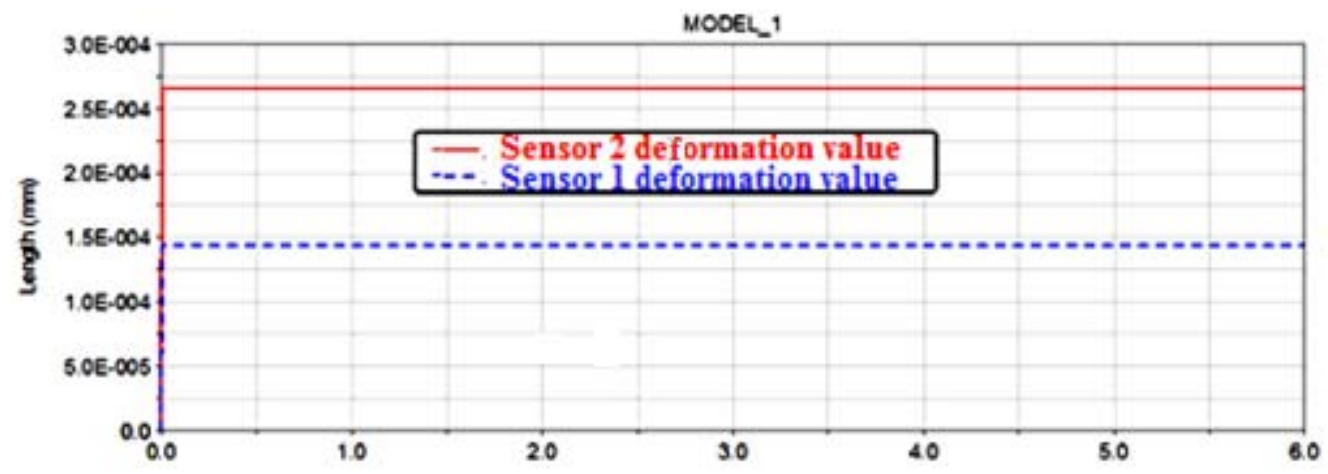

Fig.8. Sensor reading from simulation of both forces acting on the device: $F_{l}=F_{2}=50 \mathrm{~N}$.

Having two forces in perpendicular directions a similar response is observed. This leads to a conclusion that the proposed structure is suitable for measuring the input force. However, there are other forces acting on the device while performing movement. One can observe that when zoomed, the effect of inertia is visible (Fig.9). However if the value is negligibly small. 


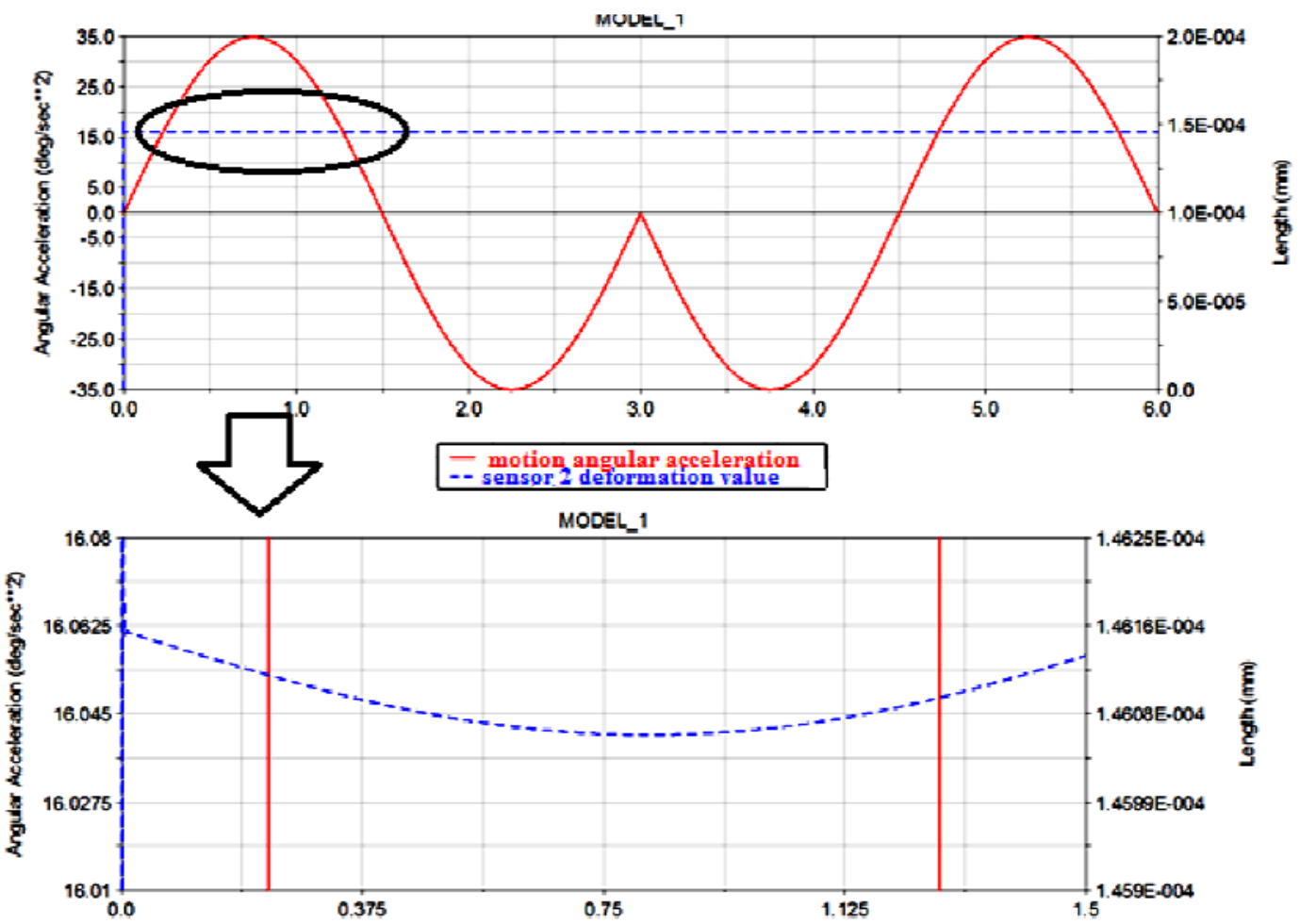

Fig.9. Sensor readings affected by the effect of inertia momentum.

\section{Concepts of control and operating modes}

Two basic modes of operation are provided for the designed system (Fig.11). The first one is the memorization and repetition of the exercise. The idea of this mode depends on the execution of one reference movement, which then will be repeated. The procedure begins with the initialization, during which the device is adapted to the dimensions of the hand. After that the specific mode of operation is selected:

- Active - in this case, the patient remains passive, while the device performs the exercise,

- Passive - the mechanism is the source of the load that must be overcome by the patient,

- Intelligent - the device, after examination of the current angular velocity, determines the adequate values of supporting or resisting forces.

In each of the above modes, the range of motion can be increased during the exercises. In this case, a physiotherapist can select the extent of the range of motion's increase and the number of repetitions that will take place.

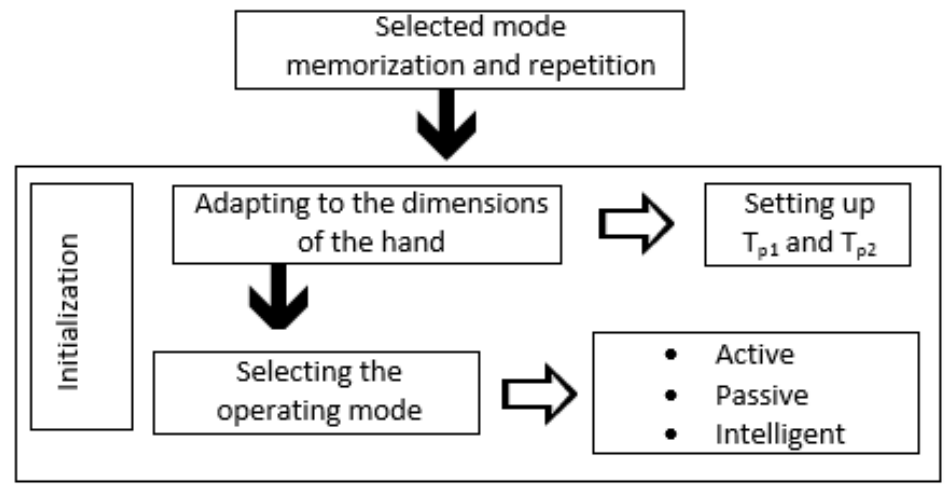

Fig.10. The idea of the initialization procedure. 
The next step is to diagnose the patient and determine basic parameters, such as the range of motion and loads. Several repetitions with increasing load should be performed, to establish the based on that disability level for each patient. This information will be recorded, thereby the progress will be visible. The next stage of this mode is to remember the exercise. The system significantly reduces torques caused by gravity, so that the patients performing the first iteration with the physiotherapist do not feel the influence of the mechanism. Memorization is to save the exercise data in the device memory: $f(t), w(t), e(t)$ for rotary actuators and $x(t), v(t), a(t)$ for linear drives.

The most important part is the repetition. The system gradually increases the torque on the drive in order to accurately repeat the exercise. This parameter is adjusted in each subsequent repetition, using the data gathered in previous cycles.

The second basic mode is called the active support (Fig.11). It is characterized by the lack of knowledge about the movement, which should be performed by the patient. The idea of this mode is to study the basic parameters of motion $f(t), w(t), e(t)$ and forces (forces directions) that the patient exerts. The system tries to predict the intentions of the patient and depending on these parameters, adequately assists or resists the movement.

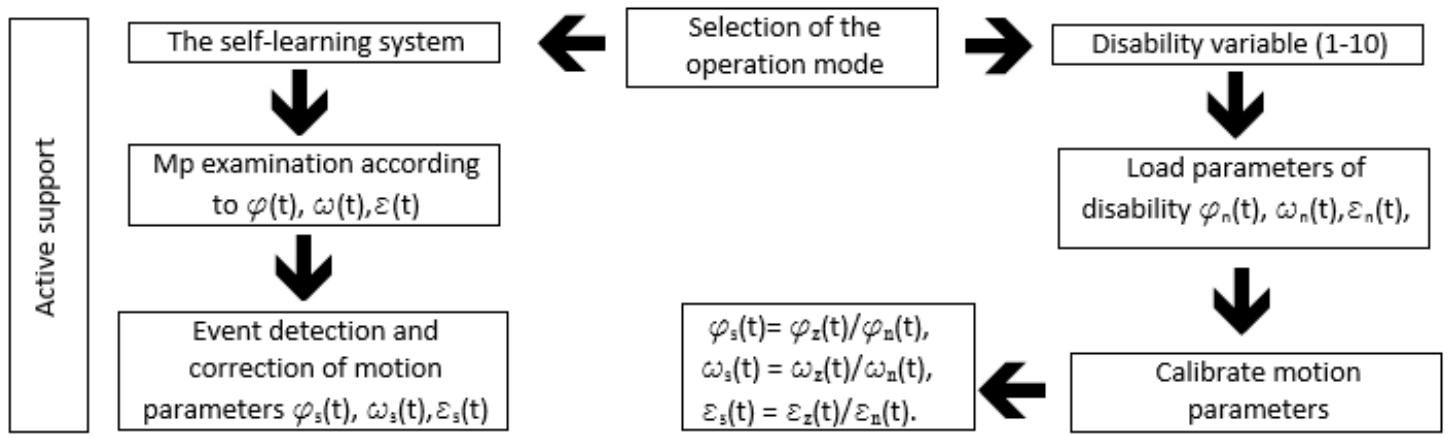

Fig.11. The idea of active support mode.

In this case there are two possibilities of specific operation: a self-learning system and a system based on parameter corresponding to the patient's disability. The former is the study of the motion generated by the patient. Depending on the gathered motion parameters, it analyzes and corrects the torque: assists or resists the movement. Data from every motion are used to assist in subsequent cycles in order to obtain greater effectiveness of support. Based on motion parameters and torque, the system is detects events such as: the end of the movement cycle, the end of the movement range, the patient's low acting force, and others. Next a decision between support and resist is made. In the second mode, the physiotherapist determines the parameters related to the disability of the patient. On this basis motion parameters and ranges are calibrated. The system decides on supporting or resisting as in the previous mode.

The third operation mode, which will be improved in the near future, is the additional simulation of real objects in a virtual environment. This would give an opportunity to make the exercises more real. Adding acoustic, haptic and visual feedback and placing the patient in a virtual environment would greatly improve his motivation. Another concept to be studied in future concept is to develop video games in this virtual environment, which will improve the patient's performance, range of motion, agility, force, etc.

\section{Conclusions}

After an analysis of the upper limb, a simplified mechanical model was determined and created. Next, the design assumptions were specified. On this basis, it was decided to design a modular construction in order to obtain a versatile device. Furthermore, the selection of the exoskeleton as a type of the design was 
dictated by the need of adjusting the device's axes of rotation to the similar axes in the human body. The assumptions were fulfilled in the design and the next step was to conduct research on the device. First, a model was built in Inventor and then its parts were imported to the MSC Adams system. The computational model was used to simulate the device work and movements providing data about its dynamics. The influence of the moment of inertia, in relation to a reduced torque at the motor shaft, is the larger, the smaller force the patient exerts. The grip construction was investigated to measure the patient's force. The simulation results proved that the new grip construction is suitable for that task. The inertia effect acting on the device while moving is negligible small. The model can be used in further investigation and optimization process focusing on obtaining the lightest possible structure. Conceptions of several operating modes of the device were also presented. Generally, the developed control algorithm assumes that the device will not need any additional sensors. The data provided by encoders and force sensors will ensure full control over the system.

\section{References}

[1] Hogan N., Krebs H.I., Charnarong J. and Sharon A. (1995): Inventors. - Massachusetts Institute of Technology, assignee. Interactive robotic therapist. US patent 5,466,213.

[2] Krebs H.I., Hogan N., Volpe B.T., Aisen M.L., Edelstein L. and Diels C. (1999): Overview of clinical trials with MIT-MANUS: a robot-aided neuro-rehabilitation facility. - Technol. Health Care; 7:419.

[3] Hesse S., Schmidt S. and Werner C. (2006): Machines to support motor rehabilitation after stroke: 10 years experience in Berlin. - Journal of Rehabilitation Research and Development, vol.43, No.5, pp.671-678.

[4] Krebs H.I., Celestino J., Williams D., Ferraro M., Volpe B. and Hogan N. (2004): 24 a wrist extension for MITMANUS. - Lecture Notes in Control and Information Science, vol.306, pp.377-390.

[5] Krebs H.I., SENIOR MEMBER, IEEE, Volpe B.T., Williams D., Celestino J., Charles K., Lynch D. and Neville H. (2007): Robot-aided neurorehabilitation: A robot for wrist rehabilitation. - IEEE Trans Neural Syst. Rehabil. Eng., vol.15, No.3, pp.327-335.

[6] Sanchez R.J., Jr., Wolbrecht E., Smith R., Liu J., Rao S., Cramer S., Ahman T., Bobrow J.E. and Reinkensmeyer D.J (2005): A pneumatic robot for re-training arm movement after stroke: rationale and mechanical design. Rehabilitation Robotics, ICORR, pp.500-504.

[7] Nef T., Mihelj M., Kiefer G., Perndl C., Muller R. and Riener R. (2007): ARMin - exoskeleton for arm therapy in stroke patients. - Rehabilitation Robotics, ICORR. IEEE 10th International Conference on 13-15 June 2007, pp.68 74.

[8] Jiang X., Xiong C., Sun R. and Xiong Y. (2010): Characteristics of the robotic arm of a 9-DoF upper limb rehabilitation robot powered by pneumatic muscles. - Intelligent Robotics and Applications, pp.463-474.

[9] Ertas I.H. and Patoglu V. (2010): A multi-functional rehabilitation device to assist forearm/wrist and grasp therapies. -- International Conference, EuroHaptics, Amsterdam, July 8-10, pp.283-290.

[10] Bochenek A. and Reicher M. (1978): Human Anatomy - Volume I. - National Institute of Medical Publications, Warsaw.

Received: August 12, 2016

Revised: September 14, 2016 